\title{
Retardation-effect-induced plasmon modes in a silica-core gold-shell nanocylinder pair
}

\author{
J.Y. Lu ${ }^{\text {a }}$, H.Y. Chao ${ }^{\text {a }}$, J.C. Wu ${ }^{\text {a }}$, S.Y. Wei ${ }^{\text {a }}$, Y.H. Chang ${ }^{\text {a,* }}$, S.C. Chen ${ }^{\text {b }}$ \\ ${ }^{a}$ Department of physics, National Taiwan University, Taipei, Taiwan \\ ${ }^{\mathrm{b}}$ Department of Electrical Engineering, Far East College, Taiwan
}

\section{A R T I C L E I N F O}

\section{Article history:}

Received 25 August 2009

Received in revised form

3 November 2009

Accepted 5 December 2009

Keywords:

Metals

Nanoshell

Dispersion

FDTD

Raman scattering

\begin{abstract}
A B S T R A C T
The retardation-effect-induced plasmon modes in a silica-core gold-shell nanocylinder pair is investigated by the two-dimension finite difference time domain method. We show that for light polarized perpendicular to the axis connecting the pair, the spectrum depends sensitively on the size of nanocylinder pair. As the size increases, several retardation-induced non-dipolar plasmon modes including multipolar modes appear in the spectrum and the resonance wavelength and strength of its plasmon modes can be tuned by changing separation width between the nanocylinder pair. Both extinction spectra as a function of size of the core-shell nanocylinder and near-field intensity associated with the coupling resonance modes are reported.
\end{abstract}

(c) 2009 Elsevier B.V. All rights reserved.

\section{Introduction}

The optical properties of metallic nanostructures are attractive because of their ability to enhance, emit, and modify the optical field. The dependence of their optical properties on the size, shape, and surrounding medium is an active subject of research, and recent advances in nanofabrication have enabled us to design the nanostructures with different shapes and functionalities, such as nanorices [1], nanorings [2], and nanoshells [3]. Compared to solid metallic particles, these core-shell nanostructures exhibit highly tunable plasmon modes that can be tuned over an extended wavelength range between visible and near infrared regions and the variation of the plasmon resonance wavelength is interpreted as originating from coupling of localized surface plasmon modes at the inner and outer surfaces of the core-shell structure [2,4].

For small nanoparticles where the quasi-static approximation is valid, only plasmon resonance modes containing dipole moments can be excited by the external light. Several recent studies have shown that by breaking the symmetry of a nanostructure, non-dipolar plasmon modes can be excited $[5,6]$. Another mechanism for exciting non-dipolar plasmon modes is phase retardation effect [7]. When the size of the nanostructures is larger than a quarter of the wavelength of the incident wave, the spatial retardation effect over the volume of the nanostructure should be considered. In this work, we use the 2-D finite different time domain method [8] to study the optical

\footnotetext{
* Corresponding author. Tel./fax: +886 33665102.

E-mail address: yhchang@phys.ntu.edu.tw (Y.H. Chang).
}

properties of a pair of silica-core gold-shell nanocylinders. The extinction spectra for the nanocylinder pair with different sizes and separation widths are studied in details to understand the effect of the phase retardation effect on the plasmon oscillation in a nanocylinder pair.

\section{Calculation methods}

Our FDTD simulation domain is separated into three regions from outside to inside: absorbing boundary, scattered field region, and the total field region. The perfectly matched layers are used as absorption boundary to prevent reflections of scattered waves back into the simulation domain. The FDTD calculations were done accurately using a mesh size of $0.25 \mathrm{~nm}$, a time step of $4.16666 \mathrm{e}-19 \mathrm{~s}$, and a Courant number of 0.5 . The program codes have been checked with the analytical theory [9], for a single silica-core gold-shell nanocylinder, as shown in the inset of Fig. 1. The permittivity of silica is taken as 2.1 . The optical response of gold is modeled using the three critical point pole pairs (CP3) model [10], which provides a good fit to tabulated experimental data [11]. The CP3 model can be expressed as

$\varepsilon(\omega)=\varepsilon_{\infty}+\frac{\sigma / \varepsilon_{0}}{i \omega}+\sum_{p=1}^{3}\left(\frac{A_{p} \Omega_{p} e^{i \phi_{p}}}{\Omega_{p}-\omega-i \Gamma_{p}}+\frac{A_{p} \Omega_{p} e^{-i \phi_{p}}}{\Omega_{p}+\omega+i \Gamma_{p}}\right)$

The value of the parameters can be found in [10]. The geometrical arrangement of the nanocylinder pair and the incident wave is shown in Fig. 1. The propagating direction of the incident wave is along the 
axis that connects the nanocylinder pair, and the electric field of the incident wave is perpendicular to this axis.

\section{Results and discussion}

\subsection{Phase-retardation-induced dipole and quadrupole plasmon modes}

We first investigate the spectral characteristics of the silicacore gold-shell nanocylinder pair by fixing the ratio of the outer radius to inner radius at $6: 5$ and the ratio of the outer radius to the inter-nanocylinders spacing at 5:1, and by changing the outer radius from 15 to $135 \mathrm{~nm}$ with a $15 \mathrm{~nm}$ interval. In Fig. 2 the simulation results show that for outer radius of $15 \mathrm{~nm}$, only plasmon modes containing electric dipole moments can be induced by incident light. This is because in this case the quasistatic approximation is valid, so that the electromagnetic field across the extent of the nanocylinder pair can be assumed to be uniform. This plasmon mode, near $720 \mathrm{~nm}$, corresponds to in-phase symmetric dipole-dipole interaction mode, and the symmetric dipole mode is a result of the electrons at the inner surface of the core-shell nanocylinder aligned symmetrically with the electrons at the outer surface [4]. For outer radius of $30 \mathrm{~nm}$, the out-of-phase symmetric dipole-dipole interaction mode, near $790 \mathrm{~nm}$, appears in the spectrum. This out-of-phase plasmon

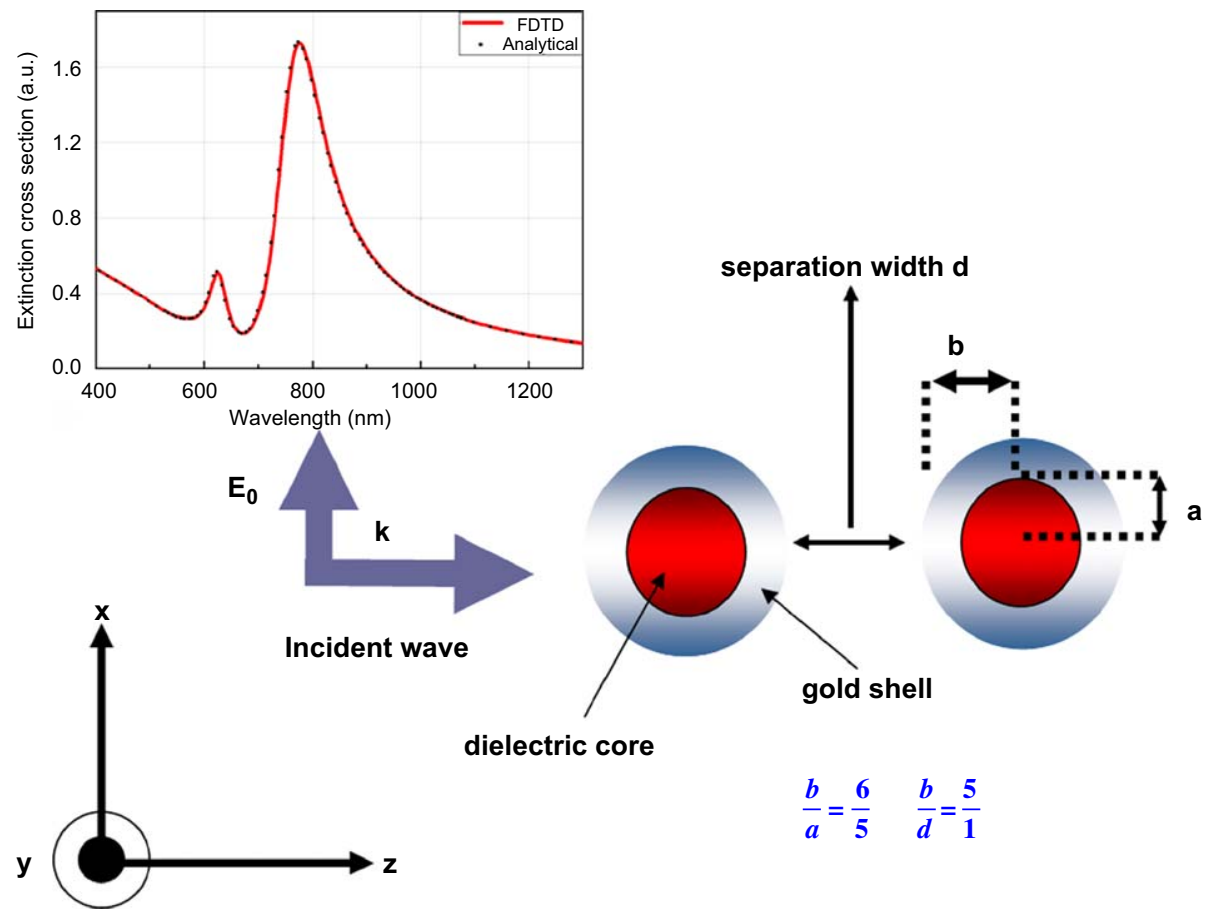

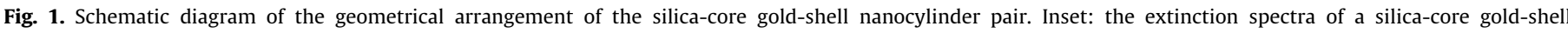

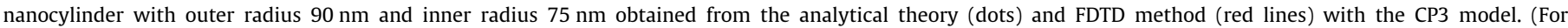
interpretation of the references to color in this figure legend, the reader is referred to the web version of this article.)
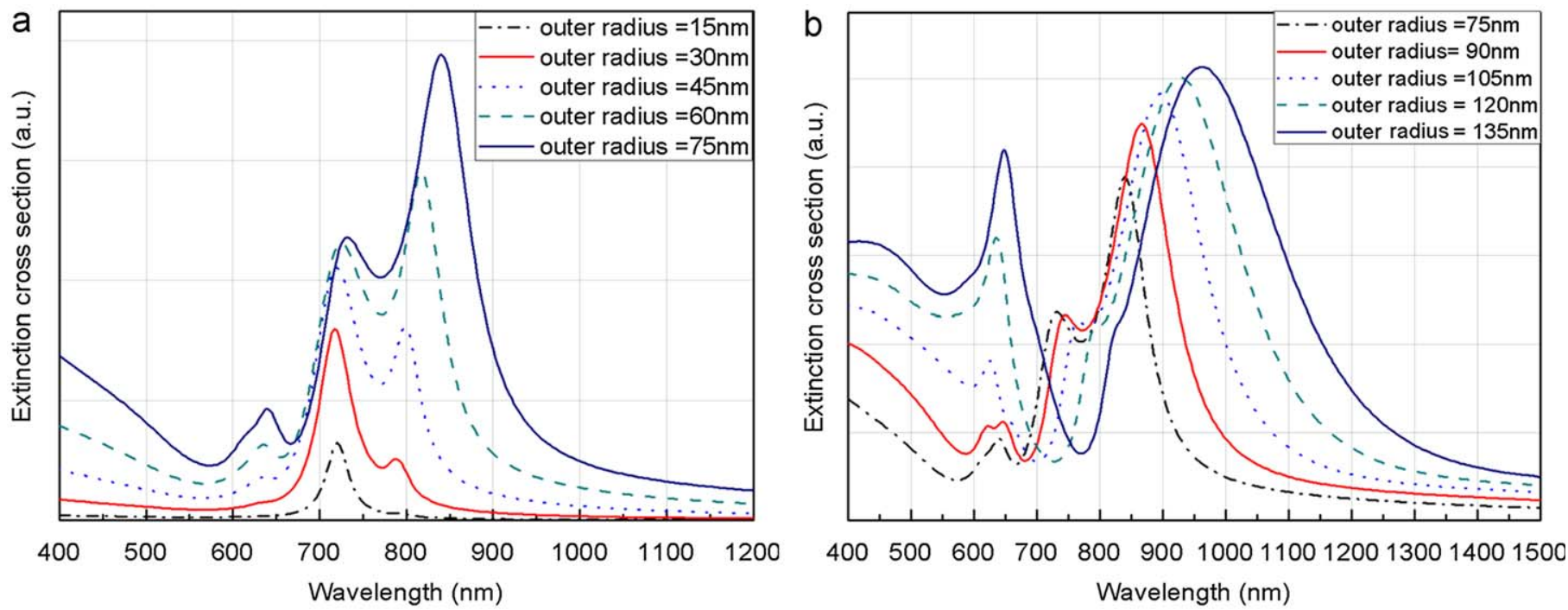

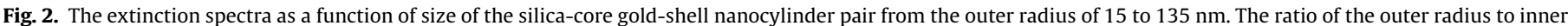
radius is fixed at $6: 5$ and the ratio of the outer radius to the inter-nanocylinders spacing at 5:1. 

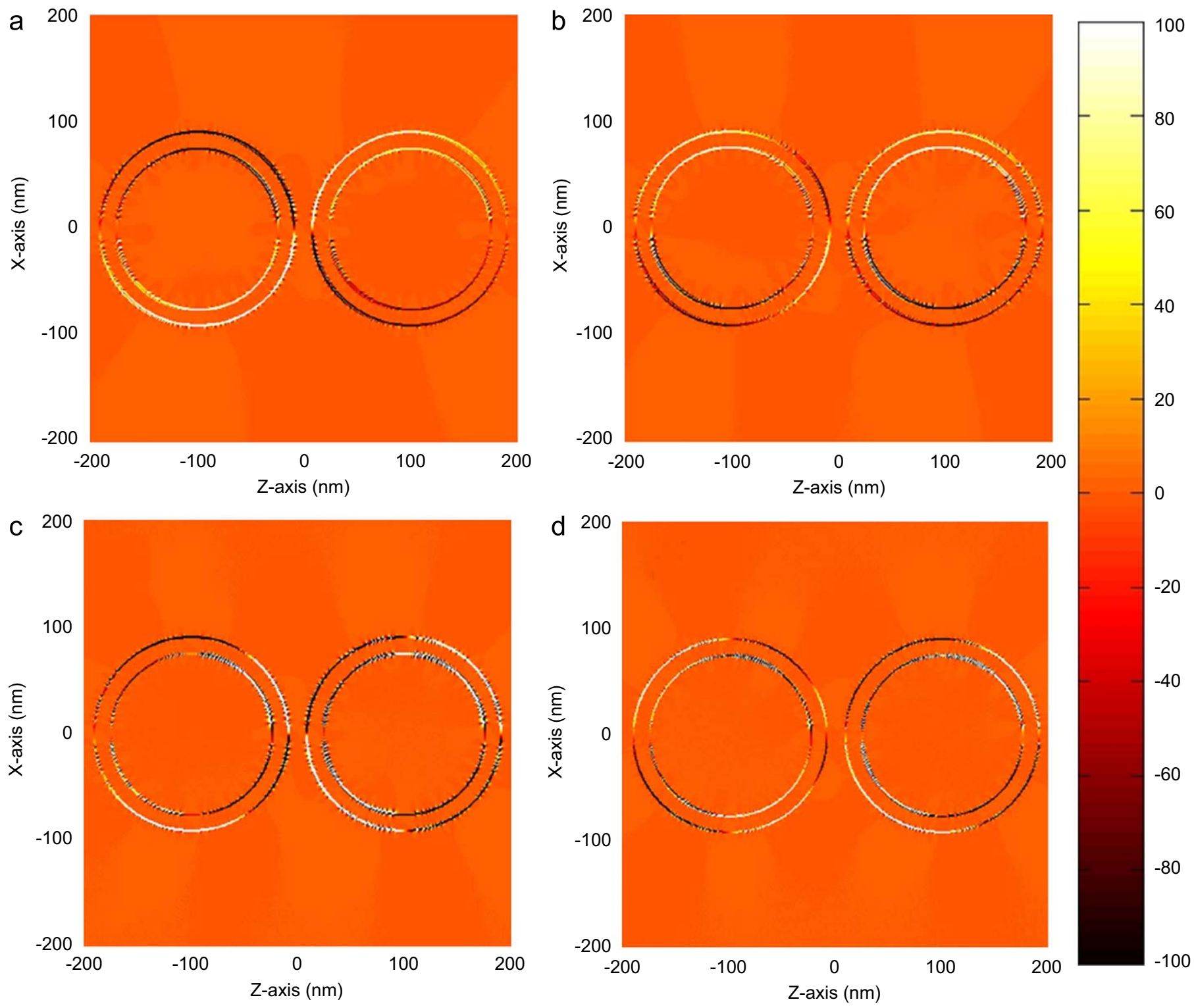

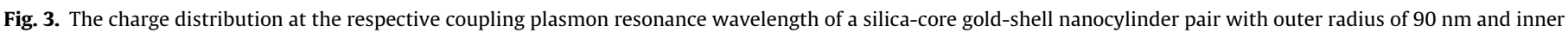
radius of $75 \mathrm{~nm}$. Wavelengths of the incident light are (a) $870 \mathrm{~nm}$, (b) $740 \mathrm{~nm}$, (c) $650 \mathrm{~nm}$, and (d) $625 \mathrm{~nm}$.

mode is excited by the phase retardation effect. The strength of this out-of-phase mode increases with the size of the nanocylinder pair. When the outer radius of the nanocylinder reaches $60 \mathrm{~nm}$, the out-of-phase symmetric dipole-dipole interaction mode becomes dominant in the extinction spectrum.

In addition to the dipole mode, the in-phase symmetric quadrupole-quadrupole interaction mode starts to appear at around $645 \mathrm{~nm}$ when the outer radius of the nanocylinder is $45 \mathrm{~nm}$. The appearance of the quadruple mode is the results of the phase retardation effect. The strength of this quadrupolequadrupole interaction mode increases as the outer radius of the nanocylinder increases. The quadrupole-quadrupole interaction mode, in addition to the in-phase mode, can also have out of phase mode. For outer radius of $90 \mathrm{~nm}$, the extinction spectrum exhibits a double peak structure: one mode, near $645 \mathrm{~nm}$, is the in-phase symmetric quadrupole-quadrupole interaction mode and the other mode, near $625 \mathrm{~nm}$, is the out-of-phase symmetric quadrupole-quadrupole interaction mode. Because the retardation effect enhances the out-of-phase mode over the in-phase mode, when the size of the nanocylinder increases, the strength of the out-of-phase mode grows in intensity, taking over the position originally occupied by the in-phase mode. At the end the only quadrupole-related feature that can be seen is the spectrum is the out-of-phase quadrupole-quadrupole interaction mode.

\subsection{Energy shift in dipole-dipole interaction and quadrupole- quadrupole interaction of a nanocylinder pair}

The coupling between symmetric dipole modes of the nanocylinder pair leads to in-phase and out-of-phase symmetric dipole-dipole interaction modes. The resonance wavelengths of the out-of phase and in-phase symmetric dipole-dipole modes are red-shifted and blue-shifted as compared to the original uncouple plasmon resonance energy, respectively. However, although the coupling between the symmetric quadrupole modes also results in the in-phase blue-shifted and out-of-phase red- 
shifted quadrupole-quadrupole resonance wavelengths but the resonance energy shifts in in-phase and out-of-phase symmetric dipole-dipole interaction modes are opposite to that of symmetric quadrupole-quadrupole interaction modes.

The shift in the resonance energy of localized surface plasmon modes in the coupled system can be understood by considering the restoring force of the coupled system. The resonance frequency of the plasmon oscillation is determined by the restoring force arising from Coulomb attraction between electron and nuclei and is thus sensitive to the charge distribution. In order to explain how the charge distribution affects the spectral behavior of the nanocylinder we show the charge distribution for each resonance mode in Fig. 3. The charge distribution of the out-of-phase dipolar plasmon mode near $860 \mathrm{~nm}$ is shown in Fig. 3(a). We can see in this figure that because the same type of charges are distributed on the opposite sides of the two nanocylinders, the restoring force acting on a cylinder is reduced by the charges on the opposite side of another nanocylinder. This reduction in the restoring force makes the out-of-phase dipolar plasmon mode have a longer resonance wavelength than an uncoupled system. On the other hand, the resonance wavelength of the in-phase dipolar plasmon mode is blue-shifted due to the increase of the restoring force as the same type of charges are distributed on the same positions in the two nanocylinders (Fig. 3(b)). The charge distribution of the quadrupole plasmon mode, near $650 \mathrm{~nm}$, is shown in Fig. 3(c). We can see that for the in-phase quadrupole mode the restoring force acting on a cylinder is reduced by the charge distribution near the gap of the other cylinder. As a result, the plasmon resonance wavelength of the in-phase quadrupole-quadrupole interaction has a longer wavelength than the resonance wavelength of the quadrupole mode of a single core-shell nanocylinder. On the contrary, for the charge distribution in Fig. 3(d), which corresponds to the charge distribution of the out-of-phase quadruple-
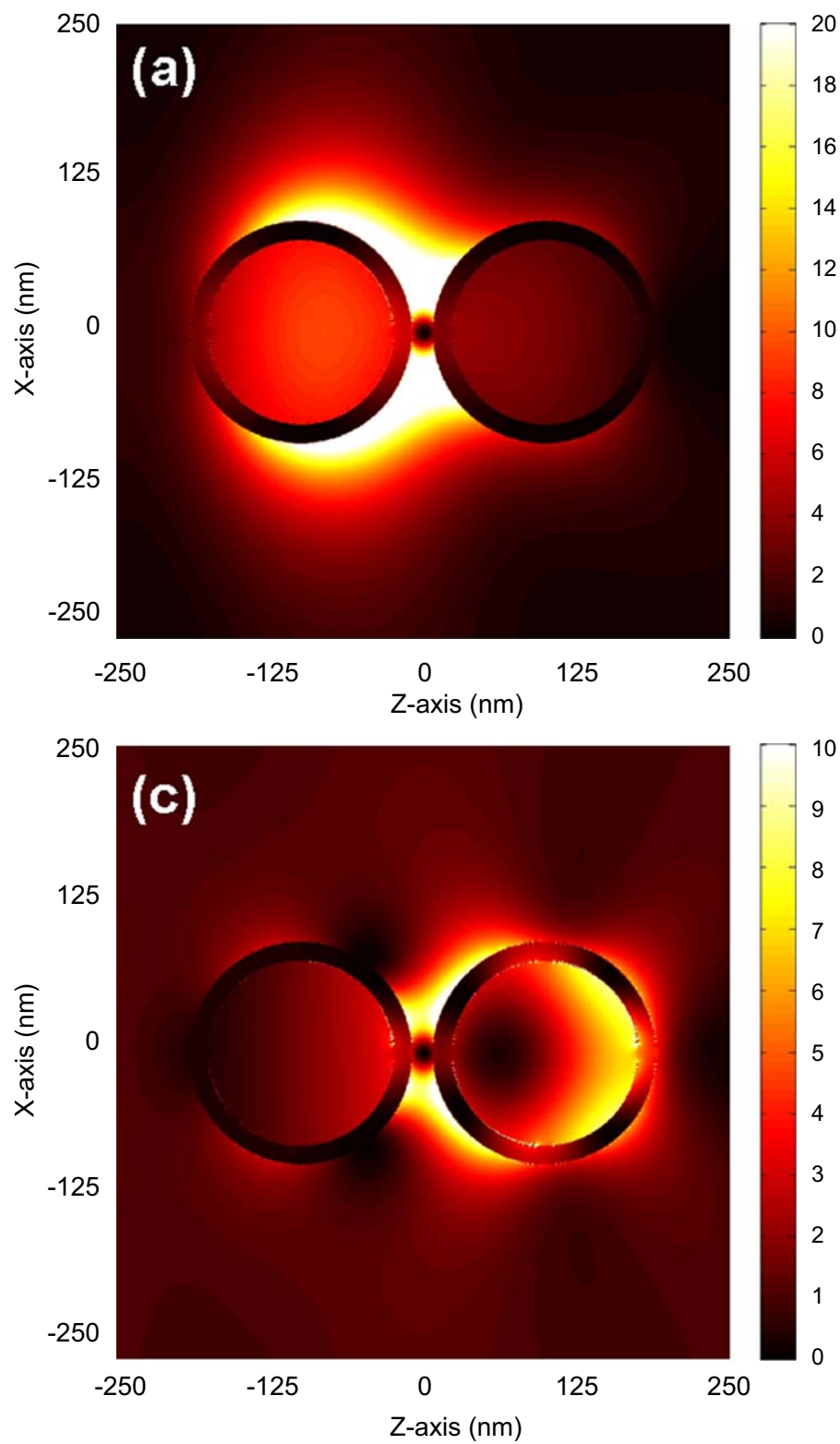
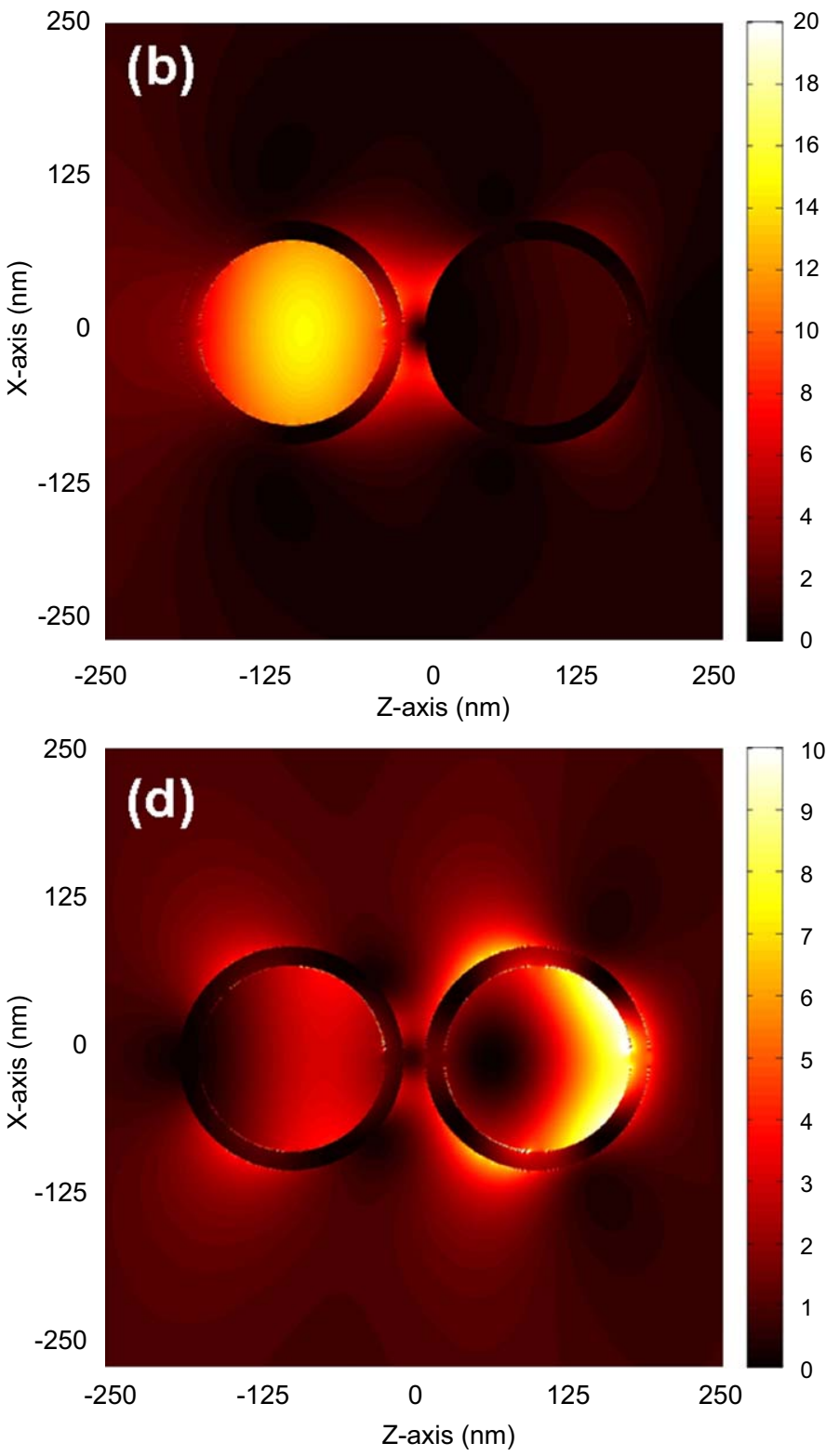

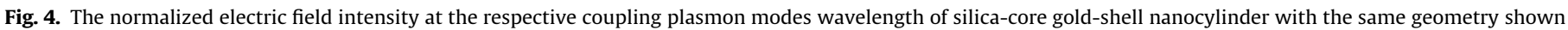
in Fig. 4. Wavelengths of the incident light are (a) $870 \mathrm{~nm}$, (b) $740 \mathrm{~nm}$, (c) $650 \mathrm{~nm}$, and (d) $625 \mathrm{~nm}$. 


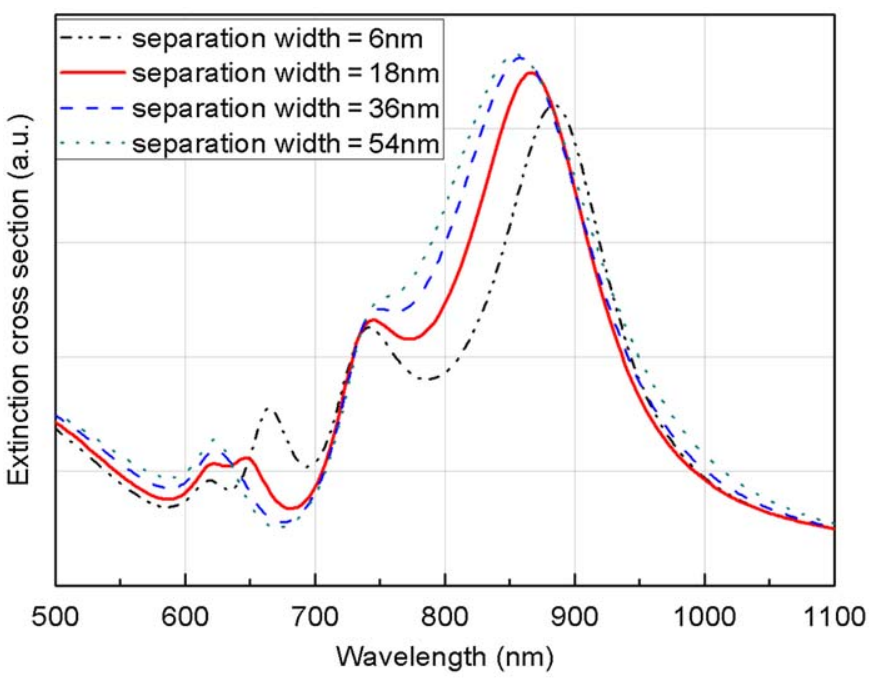

Fig. 5. The dependence of the extinction spectra on the separation width between the nanocylinder pair. The silica-core gold-shell nanocylinder has outer radius of $90 \mathrm{~nm}$ and inner radius of $75 \mathrm{~nm}$.

quadruple interaction mode, the force constant in the nanocylinder is increased. Therefore, the out-of-phase quadrupolequadrupole interaction plasmon mode has a shorter resonance wavelength, as compared to an uncoupled nanocylinder.

\subsection{The normalized electric field intensity for the respective plasmon modes}

We also investigate the normalized electric field intensity at the respective plasmon modes of a silica-core gold-shell nanocylinder pair with the same geometry as above in Fig. 4. The simulation results show that the maximum electric field intensity changes from the left-handed core-shell nanocylinder to righthanded core-shell nanocylinder when the incident wavelength decreases. It is due to the metal screening effect because the skin depth of gold increases when the wavelength of incident wave decreases. From the normalized electric field intensity distribution at the respective plasmon modes, we find that the electric field intensity concentrates in the gap between the nanocylinder pair when the charge distributions on both sides of the gap between a core-shell nanocylinder pair are opposite in sign and attract each other.

\subsection{Changing separation width between the nanocylinder pair}

The effect of the separation width between the nanocylinder pair on the plasmon resonance modes was studied and the result is shown in Fig. 5. We can find in this figure that when the separation width increases, the phase retardation effect also increases. Therefore, the strength of the out-of-phase modes, including dipole-dipole and quadrupole-quadrupole interaction modes, increases and the in-phase interaction modes becomes invisible. In addition, we find that the difference of the resonance energy between the in-phase and out-of phase interaction plasmon modes is smaller when the separation width is larger. This is because the coupling between the coreshell nanocylinder pair is reduced as the separation width is increased.

\section{Conclusion}

In conclusion, the retardation-effect-induced plasmon modes in a silica-core gold-shell nanocylinder pair are investigated by the two-dimension finite difference time domain method. The strength of the out-of-phase symmetric dipolar mode increases with the size of the nanocylinder pair and can have an intensity that is stronger than the non-retardation-effect-induced in-phase dipolar mode when the size of the nanocylinder is large enough. In addition to the dipole mode, quadrupole-quadrupole interaction mode starts to appear at around $645 \mathrm{~nm}$ when the outer radius of the nanocylinder is $45 \mathrm{~nm}$. The quadrupole-quadrupole interaction modes can have both the in-phase and out-of-phase modes. Because the retardation effect enhances the out-of-phase mode over the in-phase mode, when the size of the nanocylinder increases, the strength of the out-of-phase mode grows in intensity, taking over the position originally occupied by the inphase mode, and at the end becomes the only out-of-phase plasmon modes in the spectrum. The charge distributions in the nanocylinders for these resonance modes were studied to explain the energy shift observed in this coupled nanocylinder pair system.

\section{References}

[1] H. Wang, D.W. Brandl, F. Le, P. Nordlander, N.J. Halas, Nano Lett. 6 (2006) 827-832.

[2] J. Aizpurua, P. Hanarp, D.S. Sutherland, M. Kall, G.W. Bryant, F.J. Garcia de Abajp, Phys. Rev. Lett. 90 (2004) 057401.

[3] S.J. Oldenburg, J.B. Jackson, S.L. Wescott, N.J. Halas, Appl. Phys. Lett. 75 (1999) 2897-2899.

[4] E. Prodan, C. Radloff, N.J. Halas, P. Norlander, Science 302 (2003) 419-422.

[5] H. Wang, Y. Wu, B. Lassiter, C.L. Nehl, J.H. Hafner, P. Norlander, N.J. Halas, Proc. Natl. Acad. Sci. USA 103 (2006) 10856-10860.

[6] V.A. Fedotov, M. Rose, S.L. Prosvirnin, N. Papasimakis, N.I. Zheludev, Phys. Rev. Lett. 99 (2007) 147401.

[7] J.P. Kottmann, OJ.F. Martin, Opt. Lett. 26 (2001) 1096-1098.

[8] A. Taflove, S.C. Hagness, in: Computational Electrodynamics: The Finite Difference Time Domain Method, third ed., Artech House, 2005.

[9] H.A. Yousif, R.E. Mattis, K. Kozminski, Appl. Opt. 9 (1994) 4012-4024.

[10] Lu J.Y., Chang Y.H., Superlattices and Microstructures, 10.1016/ j.spmi.2009.07.017.

[11] P.B. Johnson, R.W. Christy, Phys. Rev. B 6 (1972) 4370-4379. 